\section{Phase-modulator-based optoelectronic oscillator for generating short optical pulse and microwave signal}

\author{
Yang Jiang, Jin-long Yu, Hao Hu, Wen-rui Wang, \\ Yao-tian Wang, and En-ze Yang \\ Tianjin University, Key Laboratory of Optoelectronics \\ Information Technical and Science, Ministry of Education, \\ School of Electronic and Information Engineering, \\ Laboratory of Fiber Optic Communication, Tianjin 300072 , \\ China
}

\begin{abstract}
A phase-modulator-based optoelectronic oscillator (OEO) is utilized to implement a self-starting optical pulse and microwave signal source. This system is able to simultaneously generate a $9.8-\mathrm{GHz}$ optical pulse stream with 141 -fs (over $100 \mathrm{~Hz}$ to $1 \mathrm{MHz}$ ) timing jitter and 6.5-ps pulse width, along with a high spectral purity electrical signal that is locked to the repetition rate of the optical pulses. The measured phase noise is $-112 \mathrm{dBc} / \mathrm{Hz}$ at $10 \mathrm{kHz}$ away from carrier, and the side modes are perfectly suppressed. () 2007 Society of Photo-Optical Instrumentation Engineers. [DOI: 10.1117/1.2775962]
\end{abstract}

Subject terms: optoelectronic oscillator; phase modulation; phase noise; microwaves.

Paper 070337LR received Apr. 19, 2007; revised manuscript received Jun. 11, 2007; accepted for publication Jun. 15, 2007; published online Sep. 10, 2007.

\section{Introduction}

The optoelectronic oscillator (OEO) has attracted many researchers because it is a very useful optical and electrical microwave signal source. ${ }^{1-3}$ Recently, a few significant works for extending the concept of OEO were reported, for instance, an OEO performs as an optical pulse source without an external microwave-driving source. ${ }^{2-5}$ But the reported pulse width is around 15 to $20 \mathrm{ps}$ (at $10 \mathrm{GHz}$ ), ${ }^{3-5}$ which limits their applications in high-speed optical time division multiplexing (OTDM) systems.

Compared with these schemes, an electro-optic phase modulator (PM)-based OEO is another effective way to perform the same function. ${ }^{6,7}$ When continuous-wave (cw) light is injected in an electro-optic PM, it is deeply chirped. After an optical synthesizer or group-delay-dispersion (GDD) circuit, the chirped cw light is compressed and trains of short pulses are generated. Enhancement of linear chirp is the key to generating short pulses. Furthermore, performing amplitude modulation (AM) before PM is effective to eliminate the residual optical power in the midpoint between adjacent pulses after compression. ${ }^{6}$

We present a scheme for a phase-modulator-based dualloop OEO, which can simultaneously produce ultra-lowjitter short optical pulse streams with high repetition rate capabilities, and corresponding high spectral purity and low phase noise electrical microwave signals. A joint of a large-

0091-3286/2007/\$25.00 @ 2007 SPIE

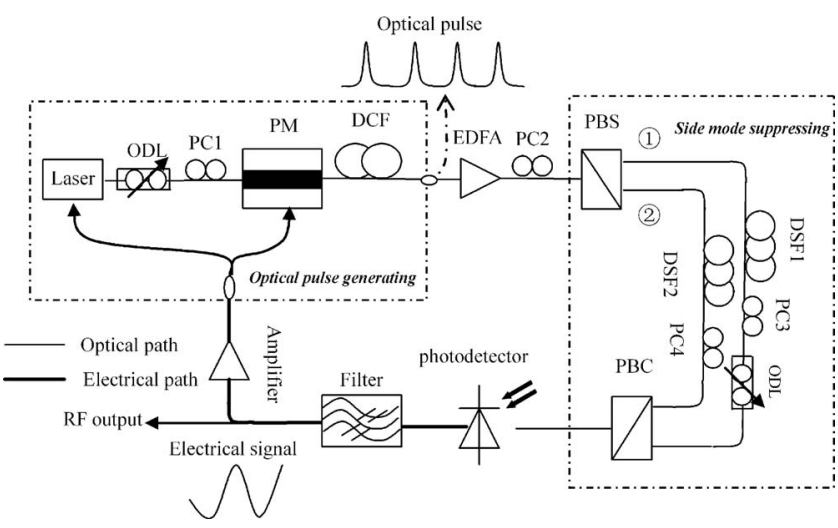

Fig. 1 The experimental setup.

signal directly modulated laser (DML) and a phase modulator is for chirp enhancing and pulse shaping. An optical domain combined dual-loop configuration is employed for side-mode suppression without adding electrical active devices. The experimental setup is depicted in Fig. 1.

\section{Principle of Operation}

As shown in Fig. 1, the system includes a directly modulated distributed feedback (DFB) laser, a $\mathrm{LiNbO}_{3}$ phase modulator with $V_{\pi}$ of $3.5 \mathrm{~V}$, an erbium-doped fiber amplifier (EDFA), a high-speed PIN photodetector, and an rf amplifier centered at $9.8 \mathrm{GHz}$ with $150-\mathrm{MHz}$ bandwidth. The electrical filter is not used due to the signal frequency already limited by the $\mathrm{rf}$ amplifier. The directly modulated light is injected into the PM after adjustment of the optical delay line (ODL) to achieve the maximum chirping rate. Polarization controller 1 ( $\mathrm{PC} 1)$ is used to obtain the best modulation. The EDFA is used for compensating the insertion loss in the system. PC2 controls the power distribution in optical path1 and path2, which are 10 and $2 \mathrm{~km}$, respectively. PC3 and PC4 tune the power distribution and correct the polarization variation caused by dispersion shift fiber (DSF). Once the oscillation threshold is reached, a stable output is achieved.

The output of large-signal DML can be approximately expressed as:

$E(t)=E_{0} \exp \left(-\frac{t^{2}}{2 t_{0}^{2}}\right) \exp \left[i\left(\omega_{0} t-\varphi(t)\right)\right]$,

where $t_{0}$ is the pulse width, which is defined as half the width of the optical pulse when amplitude decreases to 1/e of peak. $\omega_{0}$ is the angular optical frequency and $\varphi(t)$ is the phase variation depending on time $t$. When this light field is phase modulated by a sinusoidal signal of frequency $f_{m}$ with a time delay $(\Delta t)$ between DML and PM, we obtain ${ }^{7}$

$$
\begin{aligned}
E_{P M}(t)= & E_{0} \exp \left(-\frac{t^{2}}{2 t_{0}^{2}}\right) \exp \left\{i \left[\omega_{0} t-\varphi(t)\right.\right. \\
& \left.\left.-\Delta \theta \sin 2 \pi f_{m}(t+\Delta t)\right]\right\},
\end{aligned}
$$

where $\Delta \theta$ is the modulation index. Suppose $f_{m} \cdot \Delta t=k(k$ : integer), the instantaneous frequency of the modulated light is then given by 
$\nu(t)=\omega_{0} / 2 \pi-\partial \varphi(t) / 2 \pi \partial t-\Delta \theta f_{m} \cos \left(2 \pi f_{m} t\right)$.

The slope of the second term is nearly linear when it closes to the center frequency of light. In addition, near $f_{m} t$ $=n / 2+1 / 4(n$ : integer $)$, the third term gives almost a linear optical frequency chirp. Then, the extremum chirping rates can be written as

$$
(\partial \nu / \partial t)_{\max }=-\partial^{2} \varphi(t) / 2 \pi \partial t^{2} \pm 2 \pi f_{m}^{2} \Delta \theta .
$$

Because dispersion compensation fiber (DCF) can only compensate the linear negative chirps, the timing between DML and PM is controlled by an adjustable optical delay line, such that the negative chirp of the DML was gated in the PM, generating optical pulses with a flat optical frequency spectrum. The maximum chirping rate is rewritten as follows:

$(\partial \nu / \partial t)_{-\max }=-\frac{1}{2 \pi}\left|\partial^{2} \varphi(t) / \partial t^{2}\right|-2 \pi f_{m}^{2} \Delta \theta$.

Obviously, the frequency chirp is larger than that performed by DML or PM alone. After the compression with the optimum GDD, a shorter optical pulse stream is generated. For PM, a larger modulation depth is effective to gain shorter pulse, which is only limited by the endurance of the E/O crystal. Notice that the DML is not only for enhancing chirp but also for eliminating residual optical power (corresponding to the function of AM before PM), although still a minor part of energy is dispersed as the pedestal, and nonlinear chirps are formed as small peaks among pluses.
To suppress side modes, a polarization beamsplitter (PBS) and polarization beam combiner (PBC) are employed to compose an optical domain combined dual-loop structure. ${ }^{8}$ Little close-to-carrier noise is introduced, and the synthetical noise performance is greatly improved. ${ }^{8}$

Notice that DSF is used in this experiment to prevent extra GDD. It is well known that the dispersion of DCF is equal in magnitude and opposite in sign to that of the single-mode fiber (SMF). As a result, the pulses compressed by DCF are rebroadened if SMF is used. In addition, the transmission of short pulses in DSF will excite self-phase modulation, which provides the potential for further compression.

\section{Experimental Results and Conclusion}

In this experiment, breaking either optical path between PBS and PBC, it is a completed single loop OEO that can freely run when enough optical power and amplifier gain is supplied. For an electrical signal, with path 1 broken, the measured side-mode suppression ratio is $55 \mathrm{~dB}$ and mode spacing is $93.3 \mathrm{kHz}$ [see Fig. 2(a)]. With path 2 broken, the measured side-mode suppression ratio is $45 \mathrm{~dB}$ and mode spacing is $19.8 \mathrm{kHz}$ [see Fig. 2(b)]. In the dual-loop case, the side modes are perfectly suppressed [see Fig. 2(c)] and the measured phase noise is $-112 \mathrm{dBc} / \mathrm{Hz}$ at $10 \mathrm{kHz}$ [see Fig. 2(d)]. To evaluate the timing jitter $(\sigma)$ of the optical pulse stream, we integrate the spectral noise-power density from $100-\mathrm{Hz}$ to $10-\mathrm{MHz}$ offset using the following equation:
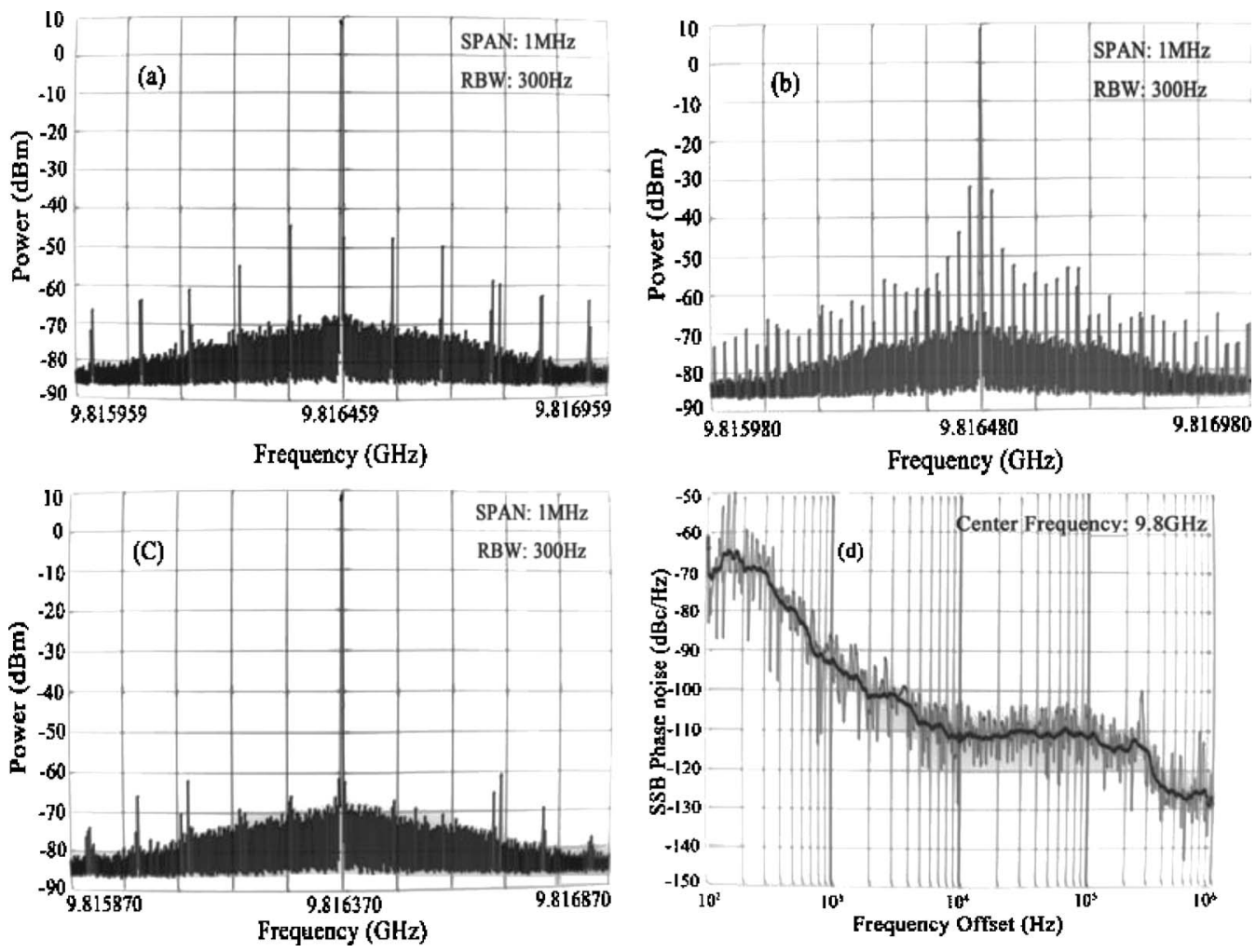

Fig. 2 Experiment results of the electrical signal: (a) the RF spectrum of signal loop OEO with 2-km loop length at SPAN $1 \mathrm{MHz}$, RBW $300 \mathrm{~Hz}$; (b) if spectrum of single loop OEO with 10-km loop length at SPAN $1 \mathrm{MHz}$, RBW $300 \mathrm{~Hz}$; (c) rf spectrum of dual-loop OEO at SPAN $1 \mathrm{MHz}$, RBW $300 \mathrm{~Hz}$; and (d) SSB phase noise spectrum of the PM-based dual-loop OEO. 

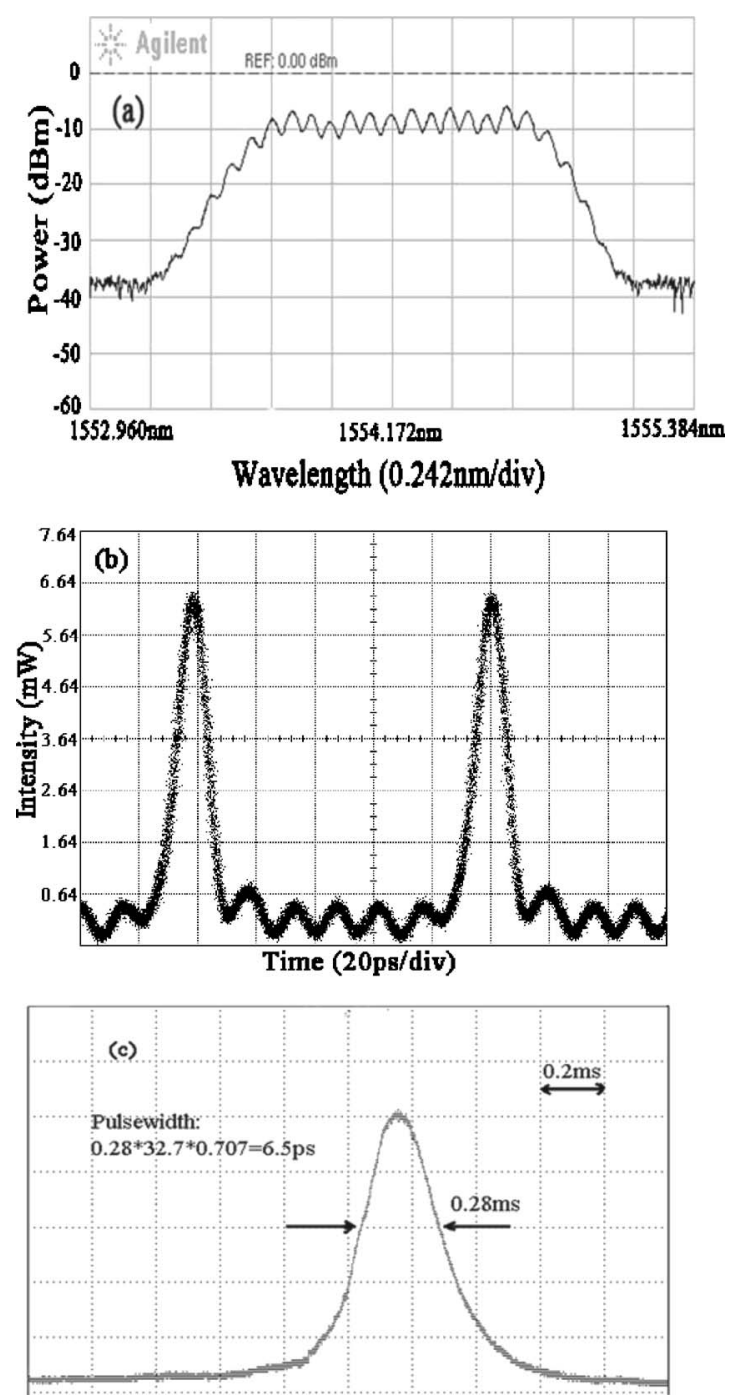

Fig. 3 Optical pulse characterization: (a) optical spectrum with 0.06-nm resolution, (b) time domain measurement of the 9.8-G optical pulse, and (c) autocorrelation trace.

$\sigma=\left[2 \int_{f_{\min }}^{f_{\max }} L(f) d f\right]^{1 / 2} / 2 \pi f_{\text {osc }}$,

where the $f_{\text {osc }}$ is the oscillation frequency. The yielding result of timing jitter is $141 \mathrm{fs}$. The phase noise and timing jitter performance is mainly influenced by MDL and EDFA, which introduce amplitude fluctuation and noise, as well as timing jitter. Furthermore, the random fiber-length fluctuations caused by environment effects are other significant factors.

Figure 3(a) shows the optical spectrum of the pulse stream. The full-width at half-maximum (FWHM) is measured to be $0.830 \mathrm{~nm}$, which is about $0.3 \mathrm{~nm}$ wider than that generated by large-signal DML alone. In this case, the modulation voltage is $3.54 \mathrm{~V}$ (corresponding to the modulation depth of $1 \pi$ ) on PM and $22.3 \mathrm{dBm}$ on DML with a bias of $50 \mathrm{~mA}$. The sampling oscilloscope trace for the optical pulse stream is given in Fig. 3(b), where the pulse width is $6.5 \mathrm{ps}$ measured by an autocorrelator [see Fig. 3(c)]. It is also clearly shown in Fig. 3(b) that the optical energy at the tail far from the pulse peak is almost perfectly eliminated. The bandwidth product of $\Delta \nu \Delta t \sim 0.65$ is not transform limited for an assumed Gaussian pulse shape, because the dispersion magnitude $(-21.68 \mathrm{ps} / \mathrm{nm})$ of DCF is not optimum and the modulated light includes uncompressible nonlinear chirps. Nevertheless, this result is better than that generated by DML or PM alone.

In conclusion, we have demonstrated a self-starting phase-modulator-based OEO for generating low-timing jitter short optical pulses and low phase noise rf signals. Pulse shaping and side-mode suppression techniques are employed in the cavity of OEO. The output is 6.5-ps-wide pulses at $9.8-\mathrm{GHz}$ repetition rate with 141 -fs timing jitter. A low phase noise, high-quality, $9.8-\mathrm{GHz}$ electrical signal is simultaneously generated that can serve as a synchronized reference in applications of this pulse source. The phase noise and timing jitter performance can be improved by controlling environment effects and using a high performance light source. In addition, the pulse rate can be directly scaled to $40 \mathrm{GHz}$ or higher by changing some optoelectronic components in the system.

\section{Acknowledgments}

The work was supported by the National Basic Research Program of China (973 Program) grant number 2003CB314900, and the National Nature Science Foundation of China under grant 60572013.

\section{References}

1. X. Yao and L. Maleki, "Optoelectronic microwave oscillator," J. Opt. Soc. Am. B 13, 1725-1735 (1996).

2. J. Lasri, P. Devgan, R. Tang, and P. Kumar, "Ultralow timing jitter $40-\mathrm{Gb} / \mathrm{s}$ clock recovery using a self-starting optoelectronic oscillator," IEEE Photonics Technol. Lett. 16, 126-128 (2004).

3. J. Lasri, P. Devgan, R. Tang, and P. Kumar, "Self-starting optoelectronic oscillator for generating ultra-low-jitter high-rate $(10 \mathrm{GHz}$ or higher) optical pulses," Opt. Express 11, 1430-1435 (2003).

4. P. Devgan, D. Serkland, G. Keeler, K. Geib, and P. Kumar, "An optoelectronic oscillator using an 850-nm VCSEL for generating low jitter optical pulses," IEEE Photonics Technol. Lett. 18, 685-687 (2006).

5. J. Lasri, A. Bilenca, D. Dahan, V. Sidorov, G. Eisenstein, D. Ritter, and K. Yvind, "Self-starting hybrid optoelectronic oscillator generating ultra low jitter $10-\mathrm{GHz}$ optical pulses and low phase noise electrical signals "IEEE Photonics Technol. Lett. 14, 1004-1006 (2002).

6. T. Kobayashi, H. Yao, K. Amano, Y. Fukushima, A. Morimoto, and T. Sueta, "Optical pulse compression using high-frequency electrooptic phase modulation," IEEE J. Quantum Electron. 24, 382-387 (1988).

7. H. Murata, A. Morimoto, T. Kobayashi, and S. Yamamoto, "Optical pulse generation by electrooptic-modulation method and its application to integrated ultrashort pulse generators," IEEE J. Sel. Top. Quantum Electron. 6, 1325-1331 (2000).

8. J. Yang, J. Yu, Y. Wang, L. Zhang, and E. Yang, "An optical domain combined dual-loop optoelectronic oscillator," IEEE Photonics Technol. Lett. 19, 807-809 (2007). 